This is the accepted manuscript of the article, which has been published in Journal of pediatric gastroenterology and nutrition, 2019, 69(4), 431-437. http://dx.doi.org/10.1097/MPG.0000000000002435

\title{
A prospective study on child morbidity and gut microbiota in rural Malawi
}

Emma Kortekangas ${ }^{1 *}$ MD, Rebecca Young ${ }^{4}$, M.Sc., Yin Bun Cheung ${ }^{2}, \mathrm{PhD}$, Yue-Mei Fan ${ }^{1}, \mathrm{PhD}$, Josh M. Jorgensen ${ }^{4}, \mathrm{PhD}$, Arox W. Kamng’ona ${ }^{3,4}, \mathrm{PhD}$, David Chaima ${ }^{5}$, M.Sc., Ulla Ashorn ${ }^{1}, \mathrm{PhD}$, Kathryn G. Dewey ${ }^{4}$, PhD, Kenneth Maleta ${ }^{5}$, PhD, Per Ashorn ${ }^{1,6}$, PhD

\section{* Corresponding author:}

Emma Kortekangas, Tampere Center for Child Health Research, Global Health, Tampere University, Faculty of Medicine and Health Technology, Arvo Ylpön katu 34, Arvo building, 33014 Tampere, Finland, Tel +358 3355 111, Fax +358 3213 4473, emma.kortekangas@tuni.fi

Conflicts of Interest and Source of Funding: This study was supported in part by a grant to the University of California Davis from the Bill \& Melinda Gates Foundation. The authors declare no conflict of interest.

The trial was registered at clinicaltrials.gov as NCT0123969

${ }^{1}$ Center for Child Health Research, Tampere University, Faculty of Medicine and Health Technology, Tampere, Finland

${ }^{2}$ Program in Health Services \& Systems Research and Centre for Quantitative Medicine, Duke-NUS Medical School, Singapore, Singapore

${ }^{3}$ Department of Biomedical Sciences, University of Malawi, College of Medicine, Blantyre, Malawi

${ }^{4}$ Program in International and Community Nutrition, University of California Davis, CA, USA

${ }^{5}$ School of Public Health and Family Medicine, University of Malawi College of Medicine, Blantyre, Malawi

${ }^{6}$ Tampere University Hospital, Department of Paediatrics, Tampere, Finland 


\begin{abstract}
Objectives: The determinants of gut microbiota composition and its effects on common childhood illnesses are only partly understood, especially in low-income settings. The aim of the present study was to investigate whether morbidity predicts gut microbiota composition in Malawian children and whether microbiota predicts subsequent morbidity. We tested the hypothesis that common infectious disease symptoms would be predictive of lower microbiota maturity and diversity.

Methods: We used data from 631 participants in a randomized-controlled nutrition intervention trial, in which a small-quantity lipid based nutrient supplement was provided to pregnant and lactating mothers and their children at 6 to 18 months of age. Fecal samples were collected from the children at $6,12,18$, and 30 months of age and analyzed using $16 \mathrm{~S}$ rRNA sequencing. Microbiota variables consisted of measures of microbiota diversity (Shannon Index), microbiota maturity (microbiota-forage Z-score), and the relative abundances of taxa. Morbidity variables included gastrointestinal and respiratory symptoms and fever.
\end{abstract}

Results: Diarrhea and respiratory symptoms from 11 to 12 months were predictive of lower microbiota-for-age Z-score and higher Shannon Index, respectively ( $\mathrm{p}=0.035$ and $\mathrm{p}=0.023$ ). Morbidity preceding sample collection was predictive of the relative abundances of several bacterial taxa at all time points. Higher microbiota maturity and diversity at 6 months were predictive of a lower incidence rate of fever in the subsequent 6 months $(\mathrm{p}=0.007$ and $\mathrm{p}=0.031)$.

Conclusions: Our findings generally do not support the hypothesis that morbidity prevalence predicts a subsequent decrease in gut microbiota maturity or diversity in rural Malawian children. Certain morbidity symptoms may be predictive of microbiota maturity and diversity and relative abundances of several bacterial taxa. Further, microbiota diversity and maturity may be associated with the subsequent incidence of fever.

Keywords: diarrhea, respiratory tract infection, fever, microbiota development, low-income countries 


\section{What is known}

- The first years of life are critical for the establishment of a healthy intestinal microbiota and are characterized by rapid changes in microbial community composition.

- The composition of the intestinal microbiota has been associated with various acute and chronic illnesses.

\section{What is new}

- Diarrhea, respiratory tract infections, and fever may influence several aspects of the microbiota composition, including bacterial taxa that change significantly during the first years of life and contribute to the maturation of the gut microbial community.

- Microbiota diversity and maturity may be associated with the subsequent incidence of fever. 


\section{Introduction}

The first years of life are critical for the establishment of a healthy intestinal microbiota and are characterized by rapid changes in microbial community composition. In young children, microbial diversity increases with age and perturbations during this period have been associated with diseases later in life.(1-5) Recent studies have reported a link between healthy intestinal microbiota development and healthy child growth, with impaired microbiota development associated with growth faltering.(6-8) Infections can also affect the microbiota composition, and the microbiota can in turn influence an individual's susceptibility to acute illnesses, including gastrointestinal and respiratory infections and malaria.(9-15) This is especially relevant in low-income settings in which infectious diseases are common and associated with malnutrition.(16,17)

The main aim of the present study was to investigate whether the prevalence of common childhood infections predicts gut microbiota composition in children. We hypothesized that frequent morbidity would be predictive of perturbed microbiota development. To study the inverse direction of association between gut microbiota and morbidity, we further assessed whether the gut microbiota is associated with subsequent morbidity. To this end, we analyzed data from children who were followed during the iLiNS-DYAD trial in Malawi. iLiNS-DYAD was a nutrition intervention trial conducted by the International Lipid-Based Nutrient Supplements Project study team (iLiNS-DYADM; NCT01239693), which studied the effect of small-quantity lipid-based nutrient supplements (LNS) on child growth.(18) The main results of the trial have been reported previously; in summary, provision of LNS to pregnant and lactating mothers and their children at 6 to 18 months of age did not promote child growth at 18 months of age.(18) Study participants were monitored closely between birth and 30 months of age and stool and other biological samples were collected at various time points. This enabled us to study the development of the gut microbiota as well as its associations with morbidity and several other potentially influential factors.

\section{Materials and Methods}

The iLiNS-DYAD trial was a randomized, controlled, and partly blinded clinical trial conducted in the Mangochi district in southern Malawi. The study enrolled 1391 pregnant women who were 
randomly assigned to receive either LNS or multiple-micronutrient tablets during pregnancy and until 6 months after giving birth, or only iron and folic acid during pregnancy and placebo until 6 months postpartum. Of the enrolled women, the first 869 were allocated to a follow-up study, during which their children received either LNS supplementation (if the mother had received LNS) or no intervention (if the mother had received iron and folic acid or multiple micronutrients) between 6 and 18 months of age. Children of these 869 women formed the study sample for the current analysis.

Clinical data were collected at weekly home visits from birth until children reached 18 months of age and fecal samples were collected at $6,12,18$, and 30 months of age. If a child had diarrhea, no fecal sample was collected and the visit was postponed by two weeks. Samples were frozen at $-20^{\circ} \mathrm{C}$ within approximately 6 hours deep frozen at $-80^{\circ} \mathrm{C}$ within 48 hours. Bacterial members of the fecal microbiota were characterized by PCR amplification of the variable V4 region of the 16S rRNA gene and sequencing of the resulting amplicons (Illumina MiSeq instrument; paired end $250 \mathrm{nt}$ reads, Illumina Inc., San Diego, CA, USA). 16S rRNA sequences were clustered into operational taxonomic units (OTUs) with at least 97\% sequence identity, and OTUs were assigned taxonomy based on the Ribosomal Database Project version 2.4 using a cutoff of $80 \%$ for the bootstrap confidence estimate.(19) To exclude artifacts, OTUs were filtered using a threshold of $0.1 \%$ of sequencing reads in at least two samples. The V4-16S sequence data generated and analyzed for this study are available through the European Nucleotide Archive under the study accession number PRJEB29433.

To measure microbiota maturity, we employed a previously described Random Forests-(RF)derived model that was generated from a reference cohort of healthy Malawian infants and children. The model was further validated among the study participants of the iLiNS-DYAD-M trial and in this cohort the Pearson correlation coefficient between chronological age and microbiota age was 0.71 $(p<0.0001) .(8)$ The sparse RF-generated model is based on the relative abundances of 25 OTUs that were most age-discriminatory. Calculated microbiota ages of study participants were compared to the median microbiota age of same-aged infants in the reference group to obtain microbiota-for-age Zscores (MAZ-scores). Details on this method have been described previously.(7,8) Microbiota diversity has been shown to increase with age during the first years of life and can thus be seen as an additional measure of microbiota maturity.(1,2) Shannon's diversity index was calculated with the 
package phyloseq in R version 3.2.1.(20) To account for unequal library sizes, OTU counts were rarefied to 5000 reads before MAZ-scores and Shannon's diversity index were calculated. To investigate associations between childhood morbidity and the relative abundances of individual bacterial taxa, unrarefied OTU counts that were normalized using cumulative sum-scaling (CSS) were included as outcomes in descriptive analyses.(21) Further, outcomes of descriptive analyses included unweighted and weighted UniFrac distances between samples as measures of phylogenetic dissimilarity.(22,23)

Morbidity variables consisted of measures of fever, diarrhea, and respiratory symptoms, which were assessed at weekly home visits between birth and 18 months. Care-givers were asked to report any illness symptoms that the child had in the previous seven days. Diarrhea was defined as three or more liquid/semi-liquid stools per day, and respiratory symptoms were defined as difficult breathing, cough, or nasal discharge in the absence of diarrhea. In addition, data collectors measured each child's temperature at each home visit, and a temperature of $37.5^{\circ} \mathrm{C}$ or above was defined as fever. These morbidity data, which were available for each day for diarrhea and respiratory symptoms and each week for fever, were used to construct several predictor variables to examine the long- and short-term influence of morbidity on microbiota. The longitudinal prevalences of diarrhea, respiratory symptoms, and fever between 0 and 18 months were used as continuous variables. Longitudinal prevalence was defined as the percentage of days with reported diarrhea or respiratory symptoms and as the percentage of weeks at which a high temperature was measured for fever.(24) In addition, dichotomized variables defined as the presence or absence of any diarrhea, respiratory symptoms, and fever, were created for the 30 days preceding fecal sample collection at 6 and 12 months.

The hypothesis that frequent morbidity is predictive of a subsequent decrease in microbiota maturity and diversity was tested using linear regression with MAZ-scores or Shannon's diversity index as a response variable and morbidity variables as predictors. In addition, the models included a measure of socioeconomic status (household assets score including information on building materials of the house, energy and water source)(25), ownership of domestic animals, water source (piped vs. any other), sanitary facility (water closet or ventilation improved pit latrine vs. none or regular pit latrine), season of fecal sample collection, maternal characteristics (age, years of education, marital 
status, and HIV status), the exact age of the child at sample collection, child sex, delivery mode, library size from 16S rRNA sequencing, and sample processing pool. Breast-feeding, household crowding, and antibiotics use as potential confounders had to be excluded from the analysis because of too many missing observations at the time points studied (>20\%). Before the main analyses, we tested for effect modification by the nutrition intervention by adding the intervention group as an interaction term to all models. Analyses were carried out in STATA version 13.

To describe specific differences in gut microbiota composition in relation to morbidity, differences in the abundances of bacterial taxa were analyzed at OTU level in STATA. Differences in relative abundances of specific OTUs were tested with multivariable zero-inflated negative binominal models to adjust for probability of zero count in relation to library size, while all the covariates aforementioned were included in the analysis. All OTUs that had non-zero counts in at least $5 \%$ of all samples were included in the analyses. To analyze whether morbidity predicts overall microbiota composition, differences in UniFrac distances were tested with permutational analysis of variance (PERMANOVA) using the adonis function in R.(26) The PERMANOVA models included all covariates and assessed the marginal effect of morbidity. Pseudo p-values were obtained through 1000 permutations.

For analyses on microbiota composition and subsequent morbidity prevalence, microbiota variables including MAZ-score, Shannon index, and relative abundances of OTUs at 6 and 12 months were used as predictor variables. Outcome variables included the longitudinal prevalence of morbidity symptoms at 6 to 12 and 12 to 18 months as continuous variables and any symptoms in the 30 days after fecal sample collection at 6 and 12 months as dichotomous variables. To analyze whether MAZscore and Shannon index predict subsequent morbidity, negative binomial regression was used for analysis of number of days ill, with an offset for the number of observation days, and logistic regression was used for dichotomous outcomes. To analyze whether relative abundances of individual OTUs predict subsequent morbidity, abundances of all OTUs that were present in at least $5 \%$ of samples were transformed to three separate dichotomized variables (zero counts vs. below median counts, below median counts vs. above median counts, and zero counts vs. above median counts) and analyzed in fully covariate-adjusted negative binominal models. 
All p-values from analyses with relative abundances of individual OTUs as predictor or outcome variables were adjusted for multiple hypothesis testing using the Benjamini-Hochberg false discovery rate (FDR) correction (q-value).

The iLiNS-DYAD study was approved by the College of Medicine Research and Ethics Committee (COMREC) and the ethics committee of the Pirkanmaa hospital district. All participants provided informed consent at enrollment by signing or thumb printing a consent form. The trial was registered at clinicaltrials.gov as NCT0123969.

\section{Results}

There were 790 live-born infants including 8 pairs of twins in the follow-up cohort of the iLiNSDYAD study. By 30 months, a total of 46 participants had not consented to extended follow-up beyond 18 months, 78 had died, and 68 were otherwise lost to follow-up. Microbiota data were available for 564 participants at 6 months, 632 at 12 months, 631 at 18 months, and 579 at 30 months. (see Figure, Supplemental Digital Content 1, http://links.lww.com/MPG/B675).

On average, children included in the analyses were born at 39.5 weeks gestation with a birth length of $49.7 \mathrm{~cm}$ and their mothers were 25 years of age at enrollment. Most did not have access to piped water or improved sanitary facilities. Compared to participants who did not provide microbiota data, the included participants were on average born at a higher gestational age, had higher length-forage Z-score at birth, and their households had a lower mean asset index and less often access to piped water (see Table, Supplemental Digital Content 2, http://links.lww.com/MPG/B676).

The mean (SD) microbiota-for-age Z-score (MAZ) was 0.77 (3.0) at 6 months, -0.24 (2.68) at 12 months, $-1.30(1.78)$ at 18 months, and $-3.70(2.55)$ at 30 months. The mean (SD) Shannon index was $1.63(0.67)$ at 6 months, $2.40(0.67)$ at 12 months, $2.95(0.62)$ at 18 months, and $3.54(0.46)$ at 30 months. The two outcome measures were strongly correlated with each other at $6(\mathrm{r}=0.66, \mathrm{p}<0.001)$, $12(\mathrm{r}=0.76, \mathrm{p}<0.001)$, and 18 months $(\mathrm{r}=0.77, \mathrm{p}<0.001)$ and moderately correlated at 30 months $(\mathrm{r}=0.44, \mathrm{p}<0.001)$.

The mean (SD) prevalence of morbidity symptoms as percentage of days with symptoms between birth and 18 months was $2.2 \%$ (3.2\%) for diarrhea and $11.5 \%$ (10.4\%) for respiratory symptoms. A 
temperature above $37.5^{\circ} \mathrm{C}$ was measured on average at $1.5 \%$ (4.1\%) of all weekly visits between birth and 18 months.

We found no evidence of effect modification by the nutrition intervention ( $\mathrm{p}>0.1$ for all interaction terms) and participants from all three intervention groups were analyzed together. Children who had any diarrhea at 11 to 12 months had on average a 0.582 (95\% CI -1.124;-0.041) lower MAZscore at 12 months than children who did not have diarrhea, and children with respiratory symptoms at 11 to 12 months had on average a 0.129 (95\% CI 0.018;0.241) higher Shannon Index at 12 months than children with no respiratory symptoms (Table 1). No associations between the longitudinal prevalence of morbidity symptoms between birth and 18 months and MAZ-score or Shannon Index at 18 or 30 months were found (see Table, Supplemental Digital Content 3, http://links.lww.com/MPG/B677).

In analyses of differences in microbiota composition at the level of individual taxa, longitudinal prevalences of fever, diarrhea, and respiratory symptoms between birth and 18 months were predictive of relative abundances of 25 OTUs at 18 and 28 OTUs at 30 months $(q<0.05)$. Any fever, diarrhea, and respiratory symptoms in the 30 days preceding sample collection was predictive of relative abundances of 64 OTUs at 6 and 95 OTUs at 12 months. All OTUs included in the analyses are listed in the Table in Supplemental Digital Content 4 (http://links.lww.com/MPG/B678) and visualized as volcano plots in Supplemental Digital Content 5 (http://links.lww.com/MPG/B679). Several associations were observed with OTUs that have been previously associated with age or growth phenotypes. Among others, diarrhea in the 30 days preceding sample collection was at 6 months negatively associated with the relative abundances of an OTU assigned to the species Bifidobacterium bifidum $(\mathrm{q}<0.001)$ and an OTU assigned to the species Bifidobacterium pseudolongum ( $\mathrm{q}=0.002)$ and positively associated with the relative abundance of an OTU assigned to the genus Ruminococcus ( $q=0.04$ ), while respiratory symptoms were positively associated with the relative abundance of an OTU assigned to the species Prevotella copri ( $q=0.01$ ) and fever was negatively associated with the relative abundance of an OTU assigned to the genus Faecalibacterium ( $q=0.04$ ). At 12 months, diarrhea preceding sample collection was positively associated with the relative abundances of OTUs assigned to the species Clostridium aldenense ( $q=0.03$ ) and Clostridium 
sordellii ( $q=0.005)$. Longitudinal prevalence of fever at 0 to 18 months was negatively associated with the relative abundance of an OTU assigned to the genus Prevotella ( $q=0.04$ ) at 18 months. (Table 2)

Diarrhea in the 30 days preceding sample collection was associated with both unweighted and weighted UniFrac distances at 6 months $(\mathrm{p}=0.002$ and $\mathrm{p}=0.001)$ and at12 months $(\mathrm{p}=0.002$ and $\mathrm{p}=0.005$ ). Additionally, respiratory symptoms were associated with unweighted UniFrac distances at 12 months ( $\mathrm{p}=0.018, \mathrm{p}=0.056$ for weighted UniFrac distance). (See Table, Supplemental Digital Content 6, http://links.lww.com/MPG/B680)

Significant associations between microbiota composition and subsequent morbidity were also observed. Higher MAZ-scores and diversity at 6 months were both predictive of less fever at 6 to 12 months. For every one-unit increase in MAZ-score at 6 months, the number of days with fever between 6 and 12 months decreased by 13\% (rate ratio [RR] 0.87, 95\% CI 0.78; 0.96), and for every one-unit increase in Shannon Index, it decreased by 33\% (RR 0.67, 95\% CI 0.47; 0.96). All results of analyses on microbiota maturity and diversity and subsequent morbidity outcomes are shown in Tables 3 and 4. In analyses with relative abundances of individual OTUs as predictors, no OTUs at 6 or 12 months were found to predict presence of fever, diarrhea, or respiratory symptoms in the 30 days after sample collection after correcting for multiple hypothesis testing.

\section{Discussion}

The present study investigated whether morbidity prevalence is predictive of future microbiota composition in a low-income setting and tested the hypothesis that frequent morbidity is associated with less mature and less diverse microbiota in children in rural Malawi. Secondary analyses identified bacterial OTUs whose relative abundances were significantly associated with previous morbidity. This study also assessed whether measures of microbiota composition are predictive of subsequent morbidity.

Diarrhea from 11 to 12 months of age was predictive of reduced microbial maturity at 12 months, and respiratory symptoms from 11 to 12 months were predictive of increased microbial diversity at 12 months. At all time-points, morbidity preceding sample collection was predictive of the relative abundances of specific bacterial taxa, several of which are part of the model used to construct MAZ- 
scores (i.e., age-discriminatory taxa) or have previously been found to be part of random forests derived models predicting lean mass or weight gain in mice (i.e., growth-discriminatory taxa).(8) UniFrac distances were associated with diarrhea preceding sample collection at 6 and 12 months and with respiratory symptoms preceding sample collection at 12 months. Higher levels of both microbiota maturity and diversity at 6 months were found to be predictive of reduced fever incidence in the subsequent 6 months. After correcting for multiple hypothesis testing, relative abundances of individual OTUs were not predictive of subsequent morbidity.

Our study had several strengths including a large sample size, an intensive and standardized follow-up of study participants, and a longitudinal study design. Morbidity data were collected weekly during the first 18 months, which enabled us to study associations between the gut microbiota and morbidity both before and after fecal sample collection. The process of fecal sample collection was designed to ensure sample quality through short transport times and timely freezing of samples after collection. However, data on diarrhea and respiratory illness prevalence were self-reported, and fever was the only morbidity variable measured by trained data collectors during weekly home visits. Our study was limited by missing data on breast-feeding, household crowding, and antibiotics use. Previous studies suggest that there is little variation in infant feeding practices in this area as almost all children are breastfed during the first 18 months of life, while exclusive breast-feeding beyond one month is very rare, but we cannot exclude potential bias by differences in antibiotic intake.(27) Because sequencing of the 16S rRNA V4 region provides low accuracy in taxonomic classification at species level, there is some uncertainty in the exact species assigned to the OTUs analyzed.(28) In addition, participants who did not provide data for this study had higher socioeconomic status than participants from whom data were analyzed, although the differences were small and unlikely to affect the validity of the results. Because the time points at which microbiota composition was examined in our study were 6 months apart, it is conceivable that short-term changes at particular ages in between those time-points were not detected.

Overall, our findings generally do not support the hypothesis that morbidity prevalence predicts a subsequent decrease in gut microbiota maturity or diversity. They do, however, suggest that diarrhea and respiratory symptoms from 11 to 12 months of age are predictive of microbiota maturity and 
diversity at 12 months of age and that morbidity prevalence may influence overall microbiota composition and relative abundances of several bacterial taxa. These include taxa that have previously been shown to be age-discriminatory in this study sample and growth-discriminatory in gnotobiotic mice colonized with the fecal microbiota of participants from this study.(8) There was no consistent pattern in differences of taxa abundances across the time points and types of symptoms studied. Microbiota diversity and maturity may also be associated with the subsequent incidence of fever in children in rural Malawi. The observed associations were different for specific morbidity symptoms which suggests that the studied symptoms cannot be thought of as one entity in terms of their relationship with the gut microbiota.

It is not clear whether morbidity is a cause or consequence of changes in microbiota composition. Moreover, the effect of morbidity on the microbiota could be mediated through increased antibiotics use, which has been shown to influence microbiota composition and an individual's susceptibility to infections.(12,29,30) Previous studies suggest that diarrhea leads to at least short-term changes in gut microbiota, which is also supported by our findings.(10,31,32) Abundances of several agediscriminatory OTUs were associated with prior diarrhea frequency. It is thus conceivable that diarrhea affects age-dependent changes in microbiota composition. Since we did not collect samples during acute episodes of diarrhea, these associations are likely not due to changes in stool consistency, which has been reported to influence microbiota composition.(33) The finding that respiratory symptoms are positively associated with microbiota diversity at 12 months is unexpected. It is possible that this is a random finding or a reflection of changes in overall microbiota composition as UniFrac distances seem to be associated with preceding respiratory symptoms. In the course of normal gut microbiota development, diversity increases rapidly around the age of 12 months. Therefore, morbidity could have a stronger influence on overall microbiota maturity and diversity at 12 months than at other time points.

Fever as measured weekly by data collectors was predictive of microbiota composition and was also predicted by microbiota maturity and diversity. This association has not been reported before; however, fever can be seen as a marker of systemic inflammation and interactions between the 
immune system and gut microbiota are well documented.(34) The gut microbiota has also previously been linked with malaria, which is a common cause of fever in the study population.(14,35)

Taken together, our results suggest that in the studied rural Malawian setting, diarrhea from 11 to 12 months of age is predictive of lower levels of microbiota maturity at 12 months, respiratory symptoms from 11 to 12 months of age are predictive of higher levels of microbiota diversity at 12 months, and higher levels of microbiota maturity and diversity at 6 months may be associated with a lower subsequent incidence of fever. Specific morbidity symptoms might be associated with changes in the relative abundances of several bacterial taxa and overall microbial community composition. There was no clear consistent pattern in the associations between microbiota and morbidity. Therefore, we cannot conclude from these findings whether a reduction in disease burden could contribute to a healthy microbiota development or whether modifications of the microbiota could reduce morbidity. Further studies on the mechanisms of interactions between pathogens, microbiota and immunity are needed to assess whether microbiota related interventions could have the potential to improve child health outcomes in this population.

\section{Acknowledgements}

We thank Laura Blanton, Michael Barratt and Nicholas Griffin from Jeffrey Gordon’s laboratory at Washington University in St. Louis for providing the 16S rRNA data sets used in these analyses. 
Table 1. Morbidity at 30 days before sample collection predicting the study participants' microbiota diversity and maturity at 6 and 12 months. Results from covariate adjusted multivariable analysis. ${ }^{a}$

\begin{tabular}{|l|l|l|l|l|}
\hline & \multicolumn{3}{|c|}{ 6 months } \\
\hline \multirow{2}{*}{ Exposure variables } & \multicolumn{2}{|c|}{ MAZ-score } & \multicolumn{2}{c|}{ Shannon Index } \\
\cline { 2 - 5 } & Coefficient (95\%CI) & P-value & Coefficient (95\%CI) & P-value \\
\hline Fever 5-6 months & $-0.4(-1.105 ; 0.304)$ & 0.265 & $-0.156(-0.353 ; 0.041)$ & 0.12 \\
\hline Diarrhea 5-6 months & $0.088(-0.403 ; 0.579)$ & 0.724 & $0.095(-0.043 ; 0.233)$ & 0.178 \\
\hline $\begin{array}{l}\text { Respiratory symptoms 5-6 } \\
\text { months }\end{array}$ & $-0.02(-0.384 ; 0.343)$ & 0.91 & $-0.035(-0.137 ; 0.068)$ & 0.505 \\
\hline
\end{tabular}

\begin{tabular}{|l|l|l|l|l|}
\hline & \multicolumn{3}{|c|}{12 months } \\
\hline \multirow{3}{*}{ Exposure variables } & \multicolumn{2}{|c|}{ MAZ-score } & \multicolumn{2}{c|}{ Shannon Index } \\
\cline { 2 - 5 } & Coefficient (95\%CI) & P-value & Coefficient (95\%CI) & P-value \\
\hline Fever 11-12 months & $0.097(-0.859 ; 1.053)$ & 0.842 & $-0.059(-0.308 ; 0.189)$ & 0.638 \\
\hline Diarrhea 11-12 months & $-0.582(-1.124 ;-0.041)$ & 0.035 & $-0.126(-0.264 ; 0.013)$ & 0.075 \\
\hline $\begin{array}{l}\text { Respiratory symptoms 11- } \\
\text { 12 months }\end{array}$ & $-0.012(-0.45 ; 0.426)$ & 0.958 & $0.129(0.018 ; 0.241)$ & 0.023 \\
\hline
\end{tabular}

MAZ-score, microbiota-for-age Z-score; CI, confidence interval

a adjusted for Household assets Z-score, ownership of any chicken, goats, and cows, source of drinking water, type of sanitary facility, maternal education, age, marital status, and HIV, child sex, delivery mode, season of sample collection, exact age at sample collection, sequencing depth, and sample processing pool 
Table 2. Time points at which age and growth discriminatory OTUs were associated with preceding morbidity

\begin{tabular}{|c|c|c|c|c|c|c|c|}
\hline \multicolumn{2}{|r|}{ Outcome } & \multicolumn{3}{|c|}{ Exposure variable } & \multicolumn{3}{|c|}{$\begin{array}{l}\text { Random forests } \\
\text { derived model }\end{array}$} \\
\hline OTU ID & Taxonomy & Fever & Diarrhea & $\begin{array}{l}\text { Respiratory } \\
\text { symptoms }\end{array}$ & Age & $\begin{array}{l}\text { Weight } \\
\text { gain }\end{array}$ & $\begin{array}{l}\text { Lean } \\
\text { mass } \\
\text { gain }\end{array}$ \\
\hline 191361 & Ruminococcus sp 51 39BFAA & & 6 months & & $\mathbf{X}$ & & \\
\hline 199145 & Faecalibacterium sp DJF VR20 & $\begin{array}{c}6 \\
\text { months }\end{array}$ & & & $\mathbf{X}$ & & \\
\hline 365385 & Bifidobacterium bifidum & & 6 months & & $\mathbf{X}$ & & \\
\hline 681370 & Bifidobacterium pseudolongum & & 6 months & & $\mathbf{X}$ & $\mathbf{X}$ & $\mathbf{X}$ \\
\hline 4436552 & Prevotella copri & & & 6 months & $\mathbf{X}$ & & \\
\hline 2148365 & Clostridium aldenense & & $\begin{array}{c}12 \\
\text { months }\end{array}$ & & & $\mathbf{X}$ & \\
\hline 242298 & Clostridium sordellii & & $\begin{array}{c}12 \\
\text { months }\end{array}$ & & & & $\mathbf{X}$ \\
\hline 4318208 & Prevotella & $\begin{array}{c}18 \\
\text { months }\end{array}$ & & & $\mathbf{X}$ & & \\
\hline
\end{tabular}

The time point at which the OTU is associated with preceding morbidity is marked in green for positive and red for negative associations. The last columns indicate whether the OTU was previously found to be part of a random forests derived model predicting age in Malawian children or weight gain or lean mass gain in mice.(8) 
Table 3. The study participants' microbiota diversity and maturity at 6 and 12 months predicting the number of days ill in the subsequent 6-month period. Results from negative binomial covariate adjusted multivariable analysis. ${ }^{a}$

\begin{tabular}{|c|c|c|c|c|c|c|}
\hline \multirow[b]{3}{*}{ Exposure variables } & \multicolumn{6}{|c|}{ 6-12 months } \\
\hline & \multicolumn{2}{|c|}{ Fever } & \multicolumn{2}{|c|}{ Diarrhea } & \multicolumn{2}{|c|}{ Respiratory symptoms } \\
\hline & RR (95\%CI) & P-value & RR (95\%CI) & P-value & RR (95\%CI) & P-value \\
\hline $\begin{array}{l}\text { MAZ-score at } 6 \\
\text { months }\end{array}$ & $0.87(0.78 ; 0.96)$ & 0.007 & $0.99(0.91 ; 1.08)$ & 0.837 & $0.97(0.92 ; 1.03)$ & 0.323 \\
\hline $\begin{array}{l}\text { Shannon Index at } 6 \\
\text { months }\end{array}$ & $0.67(0.47 ; 0.96)$ & 0.031 & $0.98(0.72 ; 1.34)$ & 0.914 & $0.97(0.78 ; 1.19)$ & 0.759 \\
\hline
\end{tabular}

\begin{tabular}{|l|l|l|l|l|l|l|}
\hline \multirow{2}{*}{ Exposure variables } & \multicolumn{3}{|c|}{ 12-18 months } & \multicolumn{3}{c|}{ Respiratory symptoms } \\
\cline { 2 - 7 } & RR (95\%CI) & P-value & RR (95\%CI) & P-value & RR (95\%CI) & P-value \\
\hline $\begin{array}{l}\text { MAZ-score at 12 } \\
\text { months }\end{array}$ & $0.96(0.90 ; 1.03)$ & 0.259 & $1.02(0.96 ; 1.09)$ & 0.427 & $1.03(0.98 ; 1.07)$ & 0.243 \\
\hline $\begin{array}{l}\text { Shannon Index at 12 } \\
\text { months }\end{array}$ & $0.9(0.7 ; 1.16)$ & 0.428 & $1.16(0.9 ; 1.5)$ & 0.261 & $1.14(0.97 ; 1.34)$ & 0.117 \\
\hline
\end{tabular}

MAZ-score, microbiota-for-age Z-score; RR, rate ratio; CI, confidence interval a adjusted for Household assets Z-score, ownership of any chicken, goats, and cows, source of drinking water, type of sanitary facility, maternal education, age, marital status, and HIV, child sex, delivery mode, season of sample collection, exact age at sample collection, sequencing depth, and sample processing pool 
Table 4. The study participants' microbiota diversity and maturity at 6 and 12 months predicting morbidity in the subsequent 30 days. Results from covariate adjusted multivariable logistic regression. ${ }^{a}$

\begin{tabular}{|l|c|c|c|c|c|c|}
\hline & \multicolumn{5}{|c|}{ 6-7 months } \\
\hline \multirow{2}{*}{ Exposure variables } & \multicolumn{2}{|c|}{ Fever } & \multicolumn{2}{c|}{ Diarnea } & \multicolumn{2}{c|}{ Respiratory symptoms } \\
\cline { 2 - 7 } & $\begin{array}{c}\text { Odds ratio } \\
(95 \% \mathrm{CI})\end{array}$ & P-value & $\begin{array}{c}\text { Odds ratio } \\
(95 \% \mathrm{CI})\end{array}$ & P-value & Odds ratio (95\%CI) & P-value \\
\hline $\begin{array}{l}0.95 \\
\text { MAZ-score at 6 } \\
\text { months }\end{array}$ & 0.731 & $\begin{array}{c}1.01 \\
(0.87 ; 1.16)\end{array}$ & 0.921 & $1.01(0.91 ; 1.12)$ & 0.880 \\
\hline $\begin{array}{l}\text { Shannon Index at 6 } \\
\text { months }\end{array}$ & $\begin{array}{c}0.66 \\
(0.24 ; 1.82)\end{array}$ & 0.419 & $\begin{array}{c}1.20 \\
(0.74 ; 1.97)\end{array}$ & 0.461 & $0.77(0.53 ; 1.12)$ & 0.170 \\
\hline
\end{tabular}

\begin{tabular}{|l|c|c|c|c|c|c|}
\hline & \multicolumn{5}{|c|}{$\mathbf{2 1 - 1 3}$ months } \\
\cline { 2 - 7 } & \multicolumn{2}{|c|}{ Fever } & \multicolumn{2}{c|}{ Diarrhea } & \multicolumn{2}{c|}{ Respiratory symptoms } \\
\cline { 2 - 7 } Exposure variables & $\begin{array}{c}\text { Odds ratio } \\
(95 \% \mathrm{CI})\end{array}$ & P-value & $\begin{array}{c}\text { Odds ratio } \\
(95 \% \mathrm{CI})\end{array}$ & P-value & Odds ratio (95\%CI) & P-value \\
\hline $\begin{array}{l}\text { MAZ-score at 12 } \\
\text { months }\end{array}$ & $0.80(0.63 ; 1.00)$ & 0.054 & $\begin{array}{c}1.06 \\
(0.96 ; 1.16)\end{array}$ & 0.259 & $0.98(0.91 ; 1.06)$ & 0.631 \\
\hline $\begin{array}{l}\text { Shannon Index at 12 } \\
\text { months }\end{array}$ & $0.47(0.22 ; 1.01)$ & 0.053 & $\begin{array}{c}1.38 \\
(0.94 ; 2.03)\end{array}$ & 0.096 & $1.10(0.83 ; 1.46)$ & 0.509 \\
\hline
\end{tabular}

MAZ-score, microbiota-for-age Z-score; CI, confidence interval

${ }^{a}$ adjusted for Household assets Z-score, ownership of any chicken, goats, and cows, source of drinking water, type of sanitary facility, maternal education, age, marital status, and HIV, child sex, delivery mode, season of sample collection, exact age at sample collection, sequencing depth, and sample processing pool 


\section{References}

1. Avershina E, Storrø O, Øien T et al. Major faecal microbiota shifts in composition and diversity with age in a geographically restricted cohort of mothers and their children. FEMS Microbiol Ecol 2014;87:280-90.

2. Yatsunenko T, Rey FE, Manary MJ et al. Human gut microbiome viewed across age and geography. Nature 2012;486:222-7.

3. Olszak T, An D, Zeissig S et al. Microbial Exposure During Early Life Has Persistent Effects on Natural Killer T Cell Function. Science 2012;336:489-93.

4. Jakobsson HE, Abrahamsson TR, Jenmalm MC et al. Decreased gut microbiota diversity, delayed Bacteroidetes colonisation and reduced Th1 responses in infants delivered by Caesarean section. Gut 2014;63:559-66.

5. Wen L, Ley RE, Volchkov PY et al. Innate immunity and intestinal microbiota in the development of Type 1 diabetes. Nature 2008;455:1109-113.

6. Smith MI, Yatsunenko T, Manary MJ et al. Gut microbiomes of Malawian twin pairs discordant for kwashiorkor. Science 2013;339:548-54.

7. Subramanian S, Huq S, Yatsunenko $\mathrm{T}$ et al. Persistent gut microbiota immaturity in malnourished Bangladeshi children. Nature 2014;510:417-21.

8. Blanton LV, Charbonneau MR, Salih T et al. Gut bacteria that prevent growth impairments transmitted by microbiota from malnourished children. Science 2016; 19;35. pii: aad3311.

9. Gorkiewicz G, Thallinger GG, Trajanoski S et al. Alterations in the Colonic Microbiota in Response to Osmotic Diarrhea. PLoS ONE 2013;8:e55817.

10. Pop M, Walker AW, Paulson J et al. Diarrhea in young children from low-income countries leads to large-scale alterations in intestinal microbiota composition. Genome Biol 2014;15:R76.

11. Nelson AM, Walk ST, Taube S et al. Disruption of the human gut microbiota following Norovirus infection. PloS One 2012;7:e48224.

12. Sekirov I, Tam NM, Jogova M et al. Antibiotic-Induced Perturbations of the Intestinal Microbiota Alter Host Susceptibility to Enteric Infection. Infect Immun 2008;76:4726-36. 
13. Hasegawa K, Linnemann RW, Mansbach JM et al. The Fecal Microbiota Profile and Bronchiolitis in Infants. Pediatrics 2016;138. pii: e20160218.

14. Yooseph S, Kirkness EF, Tran TM et al. Stool microbiota composition is associated with the prospective risk of Plasmodium falciparum infection. BMC Genomics 2015;16:631

15. Ichinohe $\mathrm{T}$, Pang IK, Kumamoto $\mathrm{Y}$ et al. Microbiota regulates immune defense against respiratory tract influenza A virus infection. Proc Natl Acad Sci U S A 2011;108:5354-9.

16. Maleta K, Virtanen SM, Espo M et al. Childhood malnutrition and its predictors in rural Malawi. Paediatr Perinat Epidemiol 2003;17:384-90.

17. Rogers GB, Ward J, Brown A et al. Inclusivity and equity in human microbiome research. The Lancet 2019;393:728-9.

18. Ashorn P, Alho L, Ashorn U et al. The impact of lipid-based nutrient supplement provision to pregnant women on newborn size in rural Malawi: a randomized controlled trial. Am J Clin Nutr 2015;101:387-97.

19. Wang Q, Garrity GM, Tiedje JM et al. Naive Bayesian Classifier for Rapid Assignment of rRNA Sequences into the New Bacterial Taxonomy. Appl Environ Microbiol 2007;73:5261-7.

20. McMurdie PJ, Holmes S. phyloseq: An R Package for Reproducible Interactive Analysis and Graphics of Microbiome Census Data. PLoS ONE 2013;8:e61217.

21. Paulson JN, Stine OC, Bravo HC et al. Differential abundance analysis for microbial marker-gene surveys. Nat Methods 2013;10:1200-2.

22. Lozupone C, Knight R. UniFrac: a new phylogenetic method for comparing microbial communities. Appl Environ Microbiol 2005;71:8228-35.

23. Lozupone C, Lladser ME, Knights D et al. UniFrac: an effective distance metric for microbial community comparison. ISME J 2011;5:169-72.

24. Morris SS, Cousens SN, Kirkwood BR et al. Is prevalence of diarrhea a better predictor of subsequent mortality and weight gain than diarrhea incidence? Am J Epidemiol 1996;144:582-8.

25. Filmer D, Pritchett LH. Estimating wealth effects without expenditure data--or tears: an application to educational enrollments in states of India. Demography 2001;38:115-32. 
26. Anderson MJ. A new method for non-parametric multivariate analysis of variance: NONPARAMETRIC MANOVA FOR ECOLOGY. Austral Ecol 2001;26:32-46.

27. Vaahtera M, Kulmala T, Hietanen A et al. Breastfeeding and complementary feeding practices in rural Malawi. Acta Paediatr Oslo Nor 1992 2001;90:328-32.

28. Gao X, Lin H, Revanna K et al. A Bayesian taxonomic classification method for $16 \mathrm{~S}$ rRNA gene sequences with improved species-level accuracy. BMC Bioinformatics 2017;18:247.

29. Korpela K, Salonen A, Virta LJ et al. Intestinal microbiome is related to lifetime antibiotic use in Finnish pre-school children. Nat Commun 2016;7:10410.

30. Gray J, Oehrle $\mathrm{K}$, Worthen $\mathrm{G}$ et al. Intestinal commensal bacteria mediate lung mucosal immunity and promote resistance of newborn mice to infection. Sci Transl Med 2017;9:eaaf9412.

31. The HC, Florez de Sessions P, Jie S et al. Assessing gut microbiota perturbations during the early phase of infectious diarrhea in Vietnamese children. Gut Microbes 2018;9:38-54.

32. Chen S-Y, Tsai C-N, Lee Y-S et al. Intestinal microbiome in children with severe and complicated acute viral gastroenteritis. Sci Rep 2017;7:46130

33. Falony G, Joossens M, Vieira-Silva S et al. Population-level analysis of gut microbiome variation. Science 2016;352:560-4.

34. Hooper LV, Littman DR, Macpherson AJ. Interactions between the microbiota and the immune system. Science 2012;336:1268-73.

35. Villarino NF, LeCleir GR, Denny JE et al. Composition of the gut microbiota modulates the severity of malaria. Proc Natl Acad Sci U S A 2016;113:2235-40. 
9310 pregnant women approached

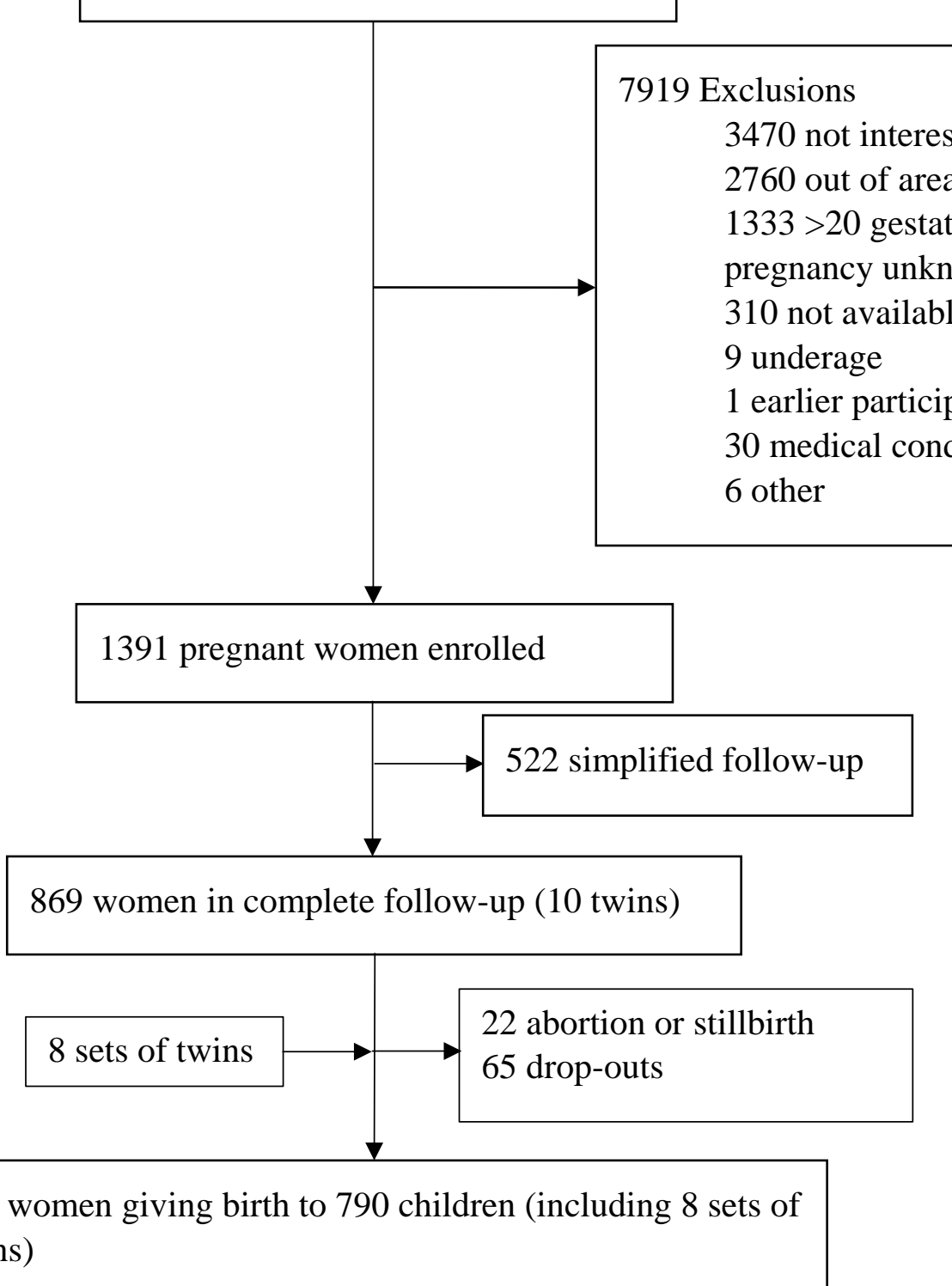

782 women giving birth to 790 children (including 8 sets of twins)

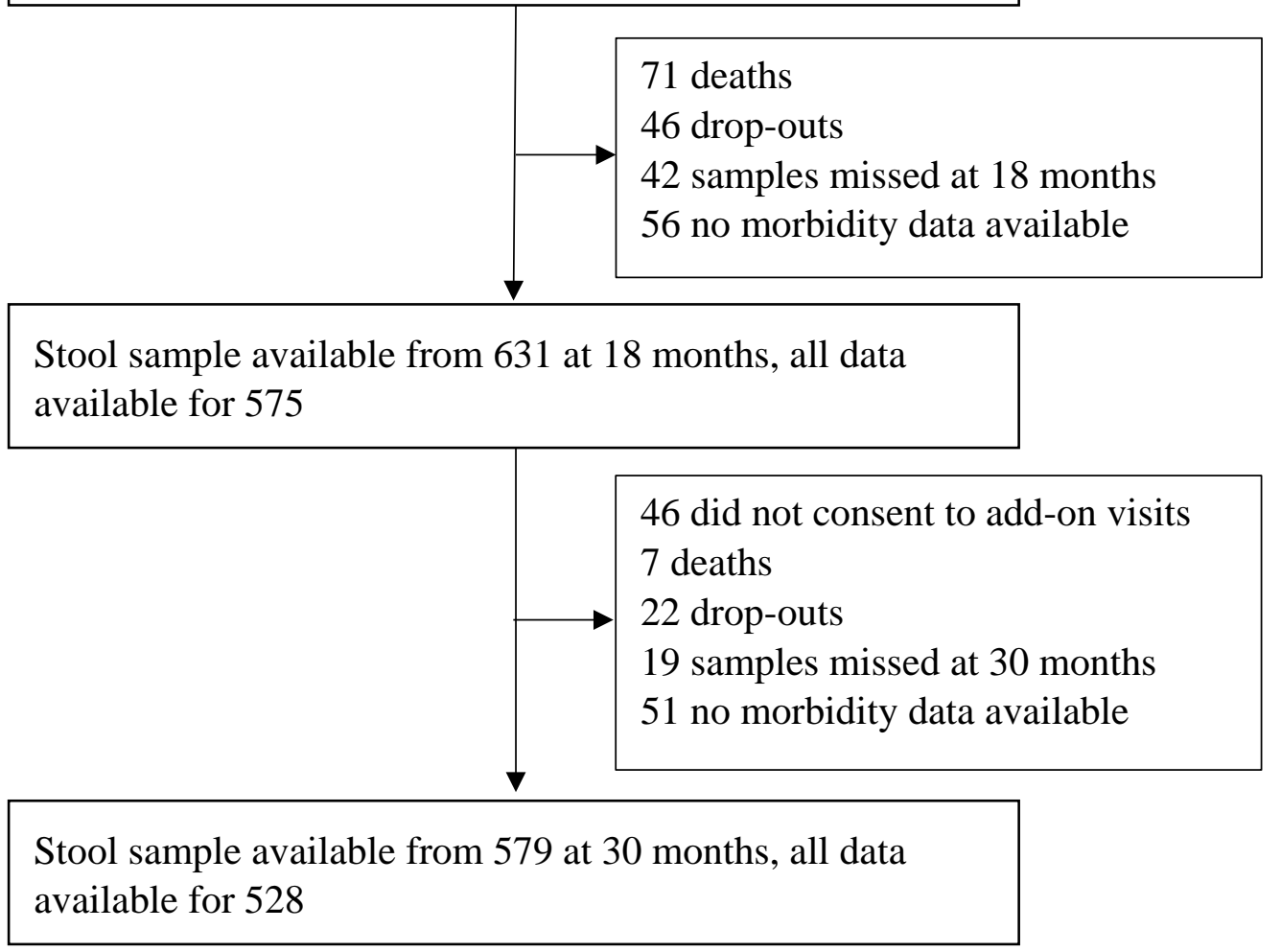




\section{Supplemental Digital Content 2. Characteristics of included and excluded study participants}

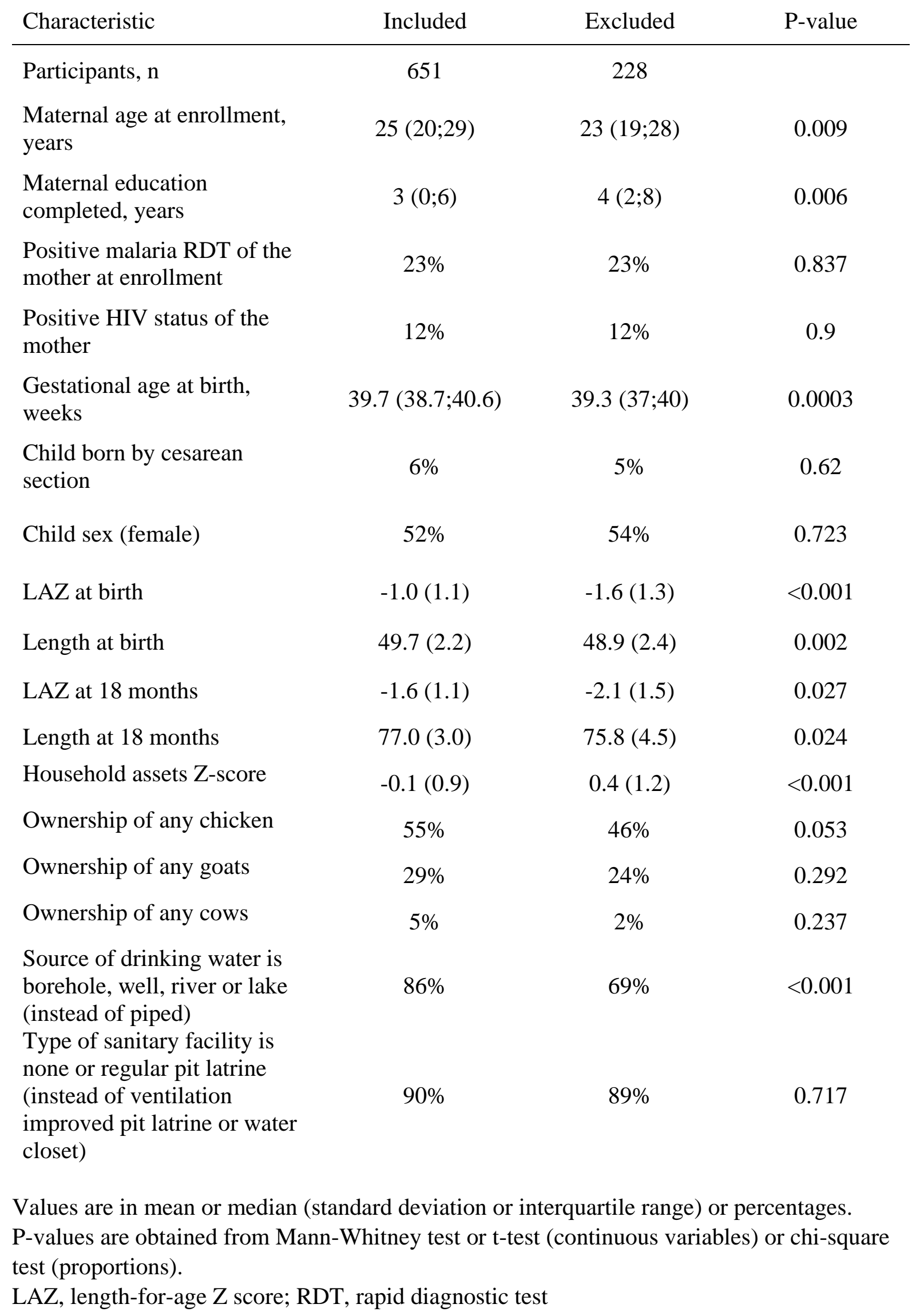


Supplemental Digital Content 3. Morbidity prevalence at 0-18 months predicting the study participants' microbiota diversity and maturity at 18 and 30 months. Results from covariate adjusted multivariable analysis. ${ }^{a}$

18 months

\begin{tabular}{|c|c|c|c|c|}
\hline \multirow[b]{2}{*}{ Exposure variables } & \multicolumn{2}{|c|}{ MAZ-score } & \multicolumn{2}{|c|}{ Shannon Index } \\
\hline & $\begin{array}{l}\text { Coefficient } \\
(95 \% \mathrm{CI})\end{array}$ & P-value & $\begin{array}{l}\text { Coefficient } \\
(95 \% \mathrm{CI})\end{array}$ & P-value \\
\hline Prevalence of fever $0-18$ months & $\begin{array}{c}-0.025 \\
(-0.11 ; 0.06)\end{array}$ & 0.565 & $\begin{array}{c}0.016 \\
(-0.013 ; 0.045)\end{array}$ & 0.284 \\
\hline $\begin{array}{l}\text { Prevalence of diarrhea } 0-18 \\
\text { months }\end{array}$ & $\begin{array}{c}-0.01 \\
(-0.061 ; 0.041)\end{array}$ & 0.703 & $\begin{array}{c}0.002 \\
(-0.015 ; 0.02)\end{array}$ & 0.810 \\
\hline \multirow[t]{3}{*}{$\begin{array}{l}\text { Prevalence of respiratory } \\
\text { symptoms } 0-18 \text { months }\end{array}$} & $\begin{array}{c}-0.003 \\
(-0.02 ; 0.015)\end{array}$ & 0.771 & $\begin{array}{c}0.002 \\
(-0.004 ; 0.007)\end{array}$ & 0.589 \\
\hline & \multicolumn{4}{|c|}{30 months } \\
\hline & \multicolumn{2}{|c|}{ MAZ-score } & \multicolumn{2}{|c|}{ Shannon Index } \\
\hline Exposure variables & $\begin{array}{l}\text { Coefficient } \\
(95 \% \mathrm{CI})\end{array}$ & P-value & $\begin{array}{c}\text { Coefficient } \\
(95 \% \mathrm{CI})\end{array}$ & P-value \\
\hline Prevalence of fever 0 -18 months & $\begin{array}{c}-0.044 \\
(-0.172 ; 0.084)\end{array}$ & 0.503 & $\begin{array}{c}0.0005 \\
(-0.022 ; 0.023)\end{array}$ & 0.964 \\
\hline $\begin{array}{l}\text { Prevalence of diarrhea } 0-18 \\
\text { months }\end{array}$ & $\begin{array}{c}-0.052 \\
(-0.128 ; 0.025)\end{array}$ & 0.184 & $\begin{array}{c}0.011 \\
(-0.002 ; 0.025)\end{array}$ & 0.097 \\
\hline $\begin{array}{l}\text { Prevalence of respiratory } \\
\text { symptoms } 0-18 \text { months }\end{array}$ & $\begin{array}{c}0.004 \\
(-0.02 ; 0.029)\end{array}$ & 0.753 & $\begin{array}{c}0.0002 \\
(-0.004 ; 0.005)\end{array}$ & 0.924 \\
\hline
\end{tabular}

MAZ-score, microbiota-for-age Z-score

a adjusted for Household assets Z-score, ownership of any chicken, goats, and cows, source of drinking water, type of sanitary facility, maternal education, age, marital status, and HIV, child sex, delivery mode, season of sample collection, exact age at sample collection, sequencing depth, and sample processing pool 

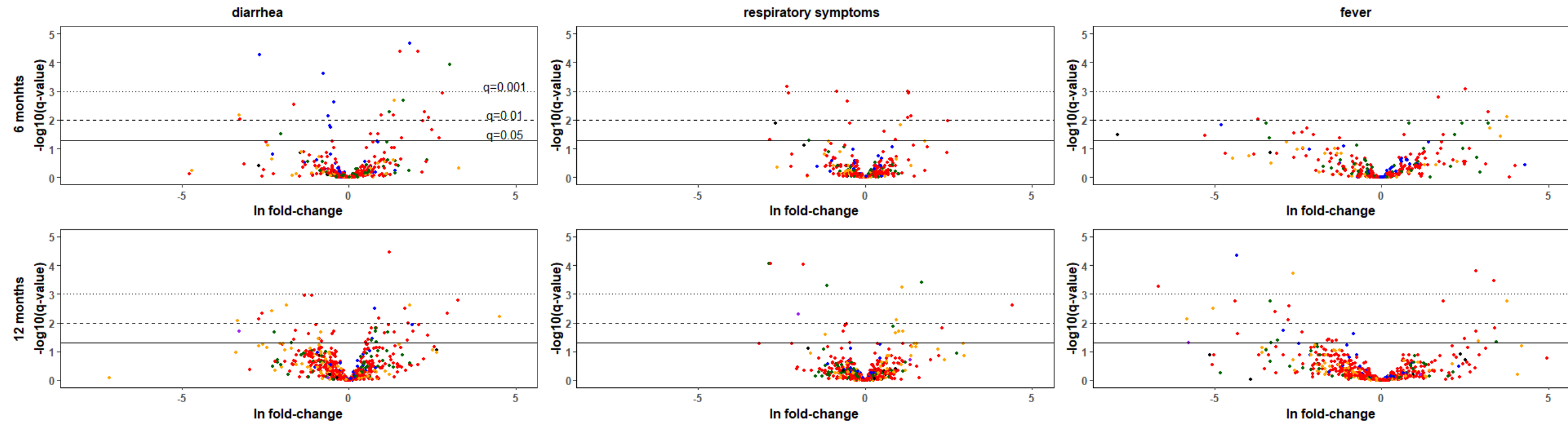

\section{Phylum}

Actinobacteria

Bacteroidetes

Elusimicrobia

Firmicutes
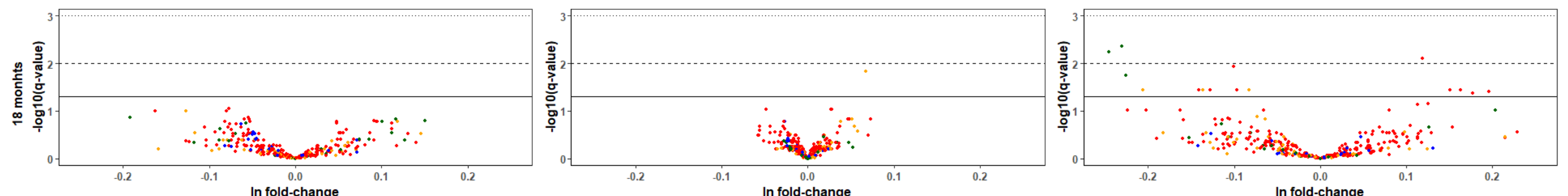

Fusobacteria

Proteobacteria

Tenericutes

Verrucomicrobia
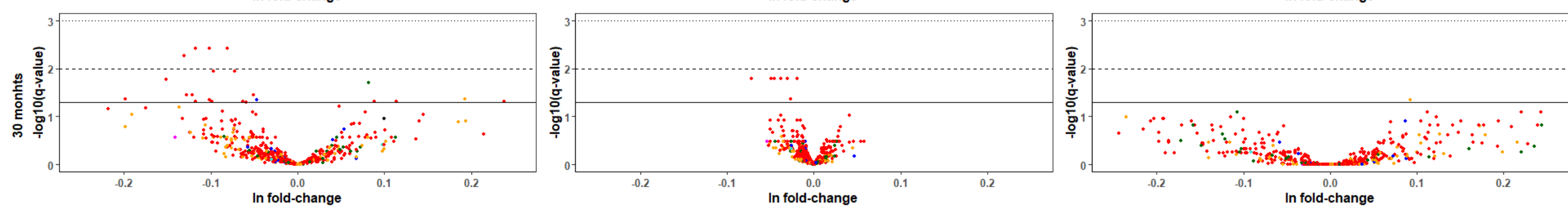

Differences in relative abundances of OTUs at 6 and 12 months predicted by any diarrhea, respiratory symptoms or fever in the 30 days preceding sample collection and differences in relative abundances of OTUs at 18 and 30 months predicted by longitudinal prevalence of diarrhea, respiratory symptoms or fever at 0 to 18 months. Ln fold-change plotted against respective q-values (fdr-corrected p-values), dots represent OTUs and are colored by phylum. Plots of 18 and 30 months data are scaled differently from 6 and 12 months data for better visualization. 


\section{Supplemental Digital Content 6. Morbidity predicting differences in UniFrac distances}

\section{6 months}

\begin{tabular}{ccccc}
\hline & \multicolumn{2}{c}{ unweighted UniFrac distance } & \multicolumn{2}{c}{ weighted UniFrac distance } \\
\cline { 2 - 5 } Exposure variables & $\mathrm{R}^{2}$-value & pseudo P-value & $\mathrm{R}^{2}$-value & pseudo P-value \\
\hline Fever 5-6 months & 0.002 & 0.625 & 0.002 & 0.392 \\
Diarrhea 5-6 months & 0.006 & 0.002 & 0.015 & 0.001 \\
Respiratory symptoms 5-6 months & 0.003 & 0.177 & 0.001 & 0.751
\end{tabular}

12 months

\begin{tabular}{ccccc}
\hline & \multicolumn{2}{c}{ unweighted UniFrac distance } & \multicolumn{2}{c}{ weighted UniFrac distance } \\
\cline { 2 - 5 } Exposure variables & $\mathrm{R}^{2}$-value & pseudo P-value & $\mathrm{R}^{2}$-value & pseudo P-value \\
\hline Fever 11-12 months & 0.002 & 0.731 & 0.001 & 0.737 \\
Diarrhea 11-12 months & 0.006 & 0.002 & 0.008 & 0.005 \\
Respiratory symptoms 11-12 & 0.004 & 0.018 & 0.004 & 0.056 \\
months & & & &
\end{tabular}

18 months

\begin{tabular}{cccccc}
\hline & \multicolumn{2}{c}{ unweighted UniFrac distance } & & \multicolumn{2}{c}{ weighted UniFrac distance } \\
\cline { 2 - 3 } Exposure variables & $\mathrm{R}^{2}$-value & pseudo P-value & & $\mathrm{R}^{2}$-value & pseudo P-value \\
\hline Fever 0-18 months & 0.002 & 0.527 & 0.003 & 0.121 \\
Diarrhea 0-18 months & 0.001 & 0.714 & 0.001 & 0.834 \\
Respiratory symptoms 0-18 & 0.001 & 0.708 & 0.001 & 0.929 \\
months & & &
\end{tabular}

30 months

\begin{tabular}{ccccc}
\hline & \multicolumn{2}{c}{ unweighted UniFrac distance } & \multicolumn{2}{c}{ weighted UniFrac distance } \\
\cline { 2 - 5 } Exposure variables & $\mathrm{R}^{2}$-value & pseudo P-value & $\mathrm{R}^{2}$-value & pseudo P-value \\
\hline Fever 0-18 months & 0.002 & 0.427 & 0.001 & 0.717 \\
Diarrhea 0-18 months & 0.002 & 0.151 & 0.001 & 0.953 \\
Respiratory symptoms 0-18 & 0.002 & 0.474 & 0.001 & 0.879 \\
months & & & &
\end{tabular}

Results from permutational analysis of variance models assessing the marginal effect of morbidity and including household assets Z-score, ownership of any chicken, goats, and cows, source of drinking water, type of sanitary facility, maternal education, age, marital status, and HIV, child sex, delivery mode, season of sample collection, exact age at sample collection, sequencing depth, and sample processing pool as covariates. Pseudo p-values were obtained through 1000 permutations. 